\title{
Coexistence of expanding abdominal aortic aneurysm and aggravated intervertebral disc extrusion -a case report-
}

\author{
Nan Seol Kim ${ }^{1}$, Sung Hyun Kang ${ }^{2}$, and Sun Young Park ${ }^{2}$ \\ Department of Anesthesiology and Pain Medicine, ${ }^{1}$ Soonchunhyang University Cheonan Hospital, Cheonan, ${ }^{2}$ Soonchunhyang \\ University Seoul Hospital, Seoul, Korea
}

\begin{abstract}
Abdominal aortic aneurysm is included in the differential diagnosis of lower back pain. Although rare, this important disease can cause potentially lethal complications. In this case, expanding abdominal aortic aneurysm coexisted with intervertebral disc extrusion. The diagnosis of abdominal aortic aneurysm was delayed, putting the patient at risk of aneurysmal rupture. In the management of patients with degenerative spinal diseases, we should not overlook the possibility of comorbidities such as an abdominal aortic aneurysm. We also suggest the importance of interpreting images more carefully, especially for elderly male patients. (Korean J Anesthesiol 2013; 65: 345-348)
\end{abstract}

Key Words: Abdominal aortic aneurysm, Differential diagnosis, Lower back pain.

Abdominal aortic aneurysm, which can be lethal, is included in the differential diagnosis of lower back pain, and this condition has been diagnosed incidentally during the investigation of other spinal pathologies [1-4]. However, because abdominal aortic aneurysm is rare, it is not normally screened for unless the patient has specific risk factors [5], which can delay the diagnosis considerably. Moreover, its coexistence with spinal diseases that cause back pain renders it more difficult to detect. We report a case in which abdominal aneurysm expansion was present combined with aggravated intervertebral disc extrusion. Since there were no suspicious clinical features, its diagnosis was delayed.

\section{Case Report}

A 73-year-old male, height $172 \mathrm{~cm}$ and weight $75 \mathrm{~kg}$, was referred to our institution after having been seen 3 months previously due to worsening lower back pain which radiated to the left buttock and thigh as well as claudication that had lasted three months. His lower back pain had been treated with caudal blocks for the last 3 years. A lumbar spine radiograph revealed degenerative scoliosis and narrowing of multiple intervertebral disc spaces. A magnetic resonance imaging (MRI) scan demonstrated marked lumbar 3-4 (L3-4) intervertebral disc extrusion, lumbar spinal stenosis, and an L3 compression fracture. He was

Received: November 15, 2012. Revised: December 18, 2012. Accepted: December 30, 2012.

Corresponding author: Sun Young Park, M.D., Department of Anesthesiology and Pain Medicine, Soonchunhyang University Seoul Hospital, 657, Hannam-dong, Yongsan-gu, Seoul 140-743, Korea. Tel: 82-2-709-9291, Fax: 82-2-790-0394, E-mail: sunnypark97@gmail.com C) This is an open-access article distributed under the terms of the Creative Commons Attribution Non-Commercial License (http:// creativecommons.org/licenses/by-nc/3.0/), which permits unrestricted non-commercial use, distribution, and reproduction in any medium, provided the original work is properly cited. 

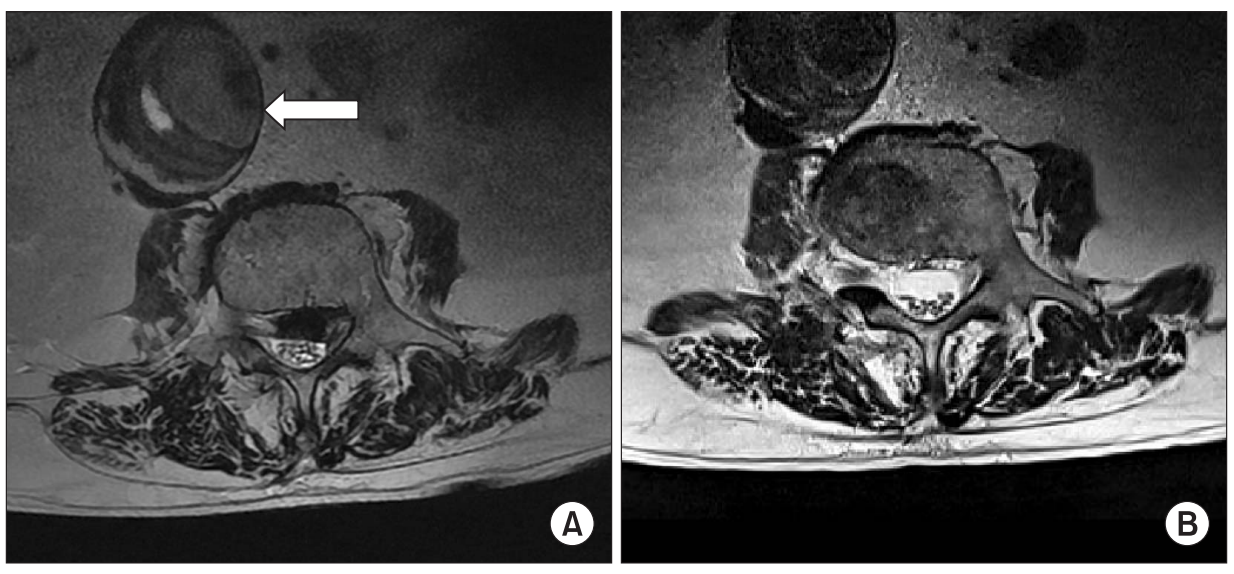

Fig. 1. Axial T2-weighted MRI. (A) At the level of L3, clearly demonstrating the aneurysmal aorta (arrow). Dilatation of the abdominal aortic aneurysm was noted. (B) Same-level axial image obtained at the first visit 3 months previously.
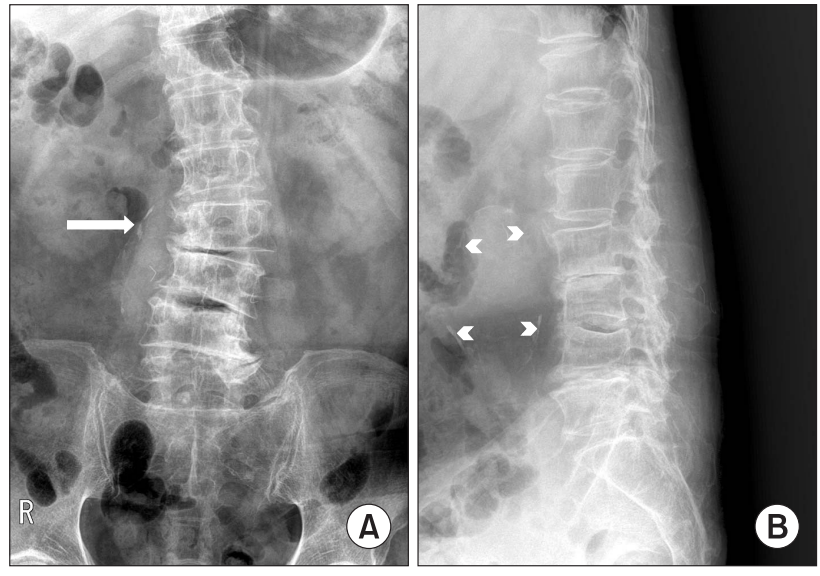

Fig. 2. Plain radiography of the lumbar spine revealed degenerative scoliosis and narrowing of multiple intervertebral spaces. (A) An AP lumbar spine radiograph showed a curvilinear atherosclerotic (calcified) lateral wall of an abdominal aortic aneurysm just lateral and right of the L3 and L4 vertebral bodies (arrows). (B) A lateral lumbar spine radiograph showed fusiform aneurysmal dilation of the abdominal aorta anterior to the L3 and L4 vertebral bodies (arrowheads).

treated with one L3-4 epidural block, medial branch blocks, and received nonsteroidal anti-inflammatory drugs. His radicular pain and claudication improved, but he complained of low back pain consistently. Therefore, a transforaminal epidural block and percutaneous epidural neuroplasty were performed. After the treatments, all symptoms improved, and only mild lower back pain which increased with walking remained. However, 2 weeks later, he was hospitalized for left leg paralysis and excruciating lower back pain that did not diminish when laying down. MRI was repeated, which identified aggravated L3-4 disc extrusion and a dilatated abdominal aortic aneurysm of nearly $6.0 \mathrm{~cm}$ (Fig. 1). Careful review of the previous MR images identified a curvilinear atherosclerotic (calcified) lateral wall of an abdominal aortic aneurysm and fusiform aneurysmal dilation of the abdominal aorta anterior to the vertebral body (Fig. 2). The patient was referred to the neurosurgery department of our hospital for further examination and treatment for the disc extrusion and abdominal aortic aneurysm.

He presented to our hospital with intractable lower back pain and pitting edema of both lower extremities. His blood pressure was $110-130 / 79-90 \mathrm{mmHg}$ and his pulse rate was $70-80 \mathrm{~min}$. Abdominal contrast enhanced computed tomography (CT) confirmed a focal lower abdominal aortic aneurysm with crescentform mural thrombus formation, without evidence of rupture (Fig. 3). A sensory conduction study and motor conduction study revealed left lumbar radiculopathy mainly of the L4, L5 nerve roots, as well as hypoesthesia on the right L4 to L5 dermatomes. Muscle strength was fair plus in left L5 innervations on manual muscle testing. The range of motion and reflexes were normal. In all extremities, the arteries were readily palpable and no pulsating mass was detected on abdominal palpation. There were no other abnormal findings on the physical examination. Bloodwork showed total cholesterol $191 \mathrm{mg} / \mathrm{dl}$, triglycerides 137 mg/dl, LDL cholesterol 137 mg/dl, HDL cholesterol $30 \mathrm{mg} / \mathrm{Dl}$ and HDL ratio 6.3. The surgeons elected to perform decompressive hemi-laminectomy and microdiscectomy on L3-L4 and transforaminal lumbar interbody fusion on L3-L4. After neurosurgery, the patient's neurologic symptoms improved except for the lower back pain. He was transferred to the thoracic surgery department and underwent endovascular aneurysm repair and aortic stent graft interposition. After surgery, his lower back pain diminished. He underwent physiotherapy for 1 month and was then discharged.

\section{Discussion}

In this case, the patient presented with lower radiating back pain, claudication, and had corresponding degenerative spinal disease. Since he had suffered from advanced degenerative spinal disease for many years, radiologists failed to notice the ab- 

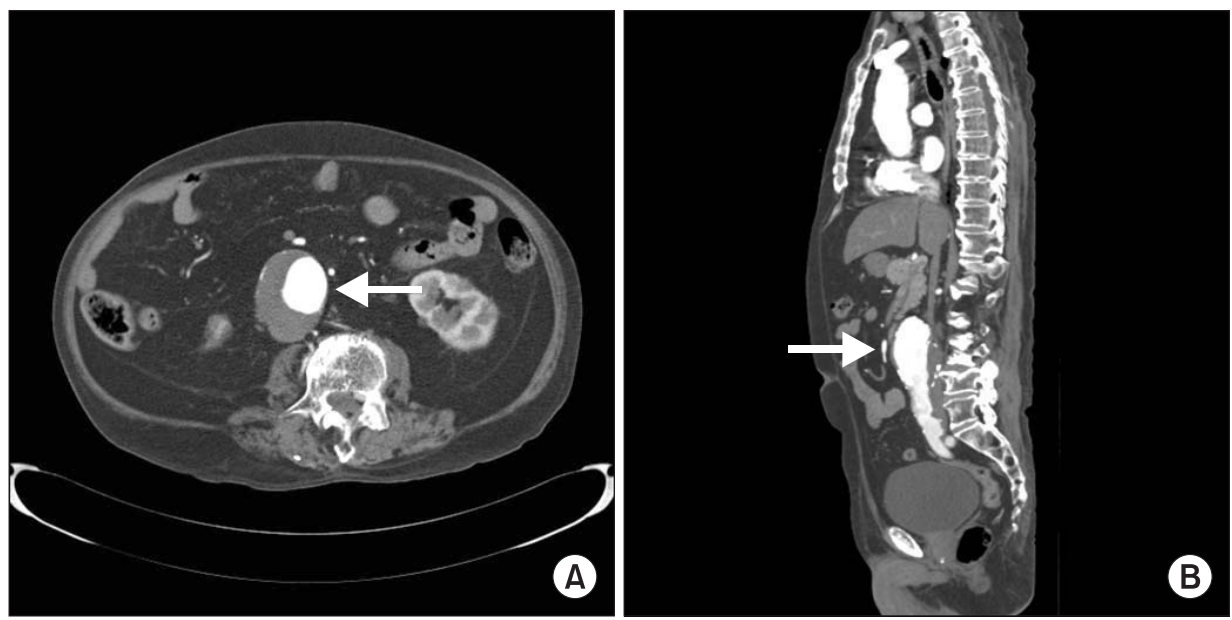

Fig. 3. Abdominal contrast enhanced computed tomography showed a focal lower abdominal aortic aneurysm with crescent-form mural thrombus formation, without evidence of rupture (arrow). The abdominal aortic aneurysm measured $4.9 \mathrm{~cm}$ (transverse diameter) by 6.1 $\mathrm{cm}$ (anteroposterior diameter) by $6.0 \mathrm{~cm}$ (length) and was infrarenal. (A) Axial view at the level of L3. (B) Lateral view.

dominal aortic aneurysm on radiographs. After aggravation of the symptoms, the expanding abdominal aortic aneurysm was finally detected on MRI and treated.

The radiating pain in the buttock \& thigh and the claudication improved after the spinal disease was treated, but the lower back pain failed to improve. Although this is a common finding in degenerative spinal disease, the fact that the lower back pain improved after vascular surgery suggests that it was due to the abdominal aortic aneurysm, as in another case [6].

Many case studies of incidental abdominal aortic aneurysm in pain clinics have been published [1-3], and abdominal aortic aneurysm is included in the differential diagnosis of lower back pain. However, this condition is rare, so is rarely screened for unless the patient has risk factors [5]. The clinical features that indicate an abdominal aortic aneurysm are cardiovascular risk factors, anticoagulants, and the lack of musculoskeletal signs [5]. Well-defined clinical risk factors include smoking, male gender, advancing age, Caucasian race, atherosclerosis, hypercholesterolemia, hypertension, and the presence a peripheral aneurysms [7-9]. In this case, the patient did not fit any of these criteria, except for gender and age. Additionally, his symptoms were apparently attributable to intervertebral disc extrusion, complicating detection of the aneurysm.

An abdominal aortic aneurysm is a degenerative condition of the aortic wall that is a segmental, full-thickness dilation of a blood vessel $>150 \%$ of its normal diameter. The majority of patients are asymptomatic. Aneurysms that produce symptoms are more likely to rupture, and the most common symptom is pain. The pain is typically located in the abdomen but can also radiate to the back, flank or groin. Complaints of new or worsening pain are suggestive of a rapidly expanding aneurysm or impending rupture [10]. The worsening back pain in this case was due to expansion of the aneurysm. Additionally, the aneurysm was approximately $6.0 \mathrm{~cm}$, and larger aneurysms are much more likely to rupture, with the risk increasing markedly for those greater than $5.5 \mathrm{~cm}$ [11]. Therefore, the patient was at a great risk of aneurysmal rupture while the diagnosis was delayed.

Trompeter and Paremain [4] reported a case series of four patients who had incidental abdominal aortic aneurysms detected on lumbosacral MRI. In half of the cases, the radiologist failed to identify the aneurysm, as in this case. The report emphasized the importance of closely examining all aspects of the images and of remembering the extraspinal causes of back and leg pain. Gouliamos et al. [12] also suggested that patients with lowback pain, above 55 years of age, who undergo lumbar spine CT, should also be screened for aortic disease by expanding the field of view sufficiently to fully visualize the entire axial diameter of the abdominal aorta. Although abdominal ultrasonography is the initial diagnostic test of choice, MRI or CT, even lateral spine plain radiographs (commonly used imaging studies for spinal diseases) are useful for assessing an abdominal aortic aneurysm [13]. The aneurysm in this case was also detectable on lateral spine plain radiographs.

Ultrasound screening studies have found that 4 to $8 \%$ of older males have an occult abdominal aortic aneurysm $[8,14,15]$. However, since the incidence of abdominal aortic aneurysm rises sharply in individuals over 60 years of age, it may be more commonly diagnosed in the current aging population.

Although the patient was at risk of aneurysmal rupture, the diagnosis of abdominal aortic aneurysm was delayed due to the coexistence of spinal pathology. When managing patients with degenerative spinal diseases, the possibility of an abdominal aortic aneurysm should not be overlooked. Although this disease is not common, it can be fatal if untreated. Therefore, we suggest the importance of careful radiologic interpretation and clinical examination, including abdominal palpation and auscultation in cases of suspicion, especially in elderly male patients. 


\section{References}

1. James J, Kuduvalli M, Lu JY, Rashid A. Coincidence of spinal canal stenosis and thoracoabdominal aortic aneurysm. Asian Cardiovasc Thorac Ann 2007; 15: 255-7.

2. Patel SN, Kettner NW. Abdominal aortic aneurysm presenting as back pain to a chiropractic clinic: a case report. J Manipulative Physiol Ther 2006; 29: 409.e1-7.

3. de Boer NJ, Knaap SF, de Zoete A. Clinical detection of abdominal aortic aneurysm in a 74-year-old man in chiropractic practice. J Chiropr Med 2010; 9: 38-41.

4. Trompeter AJ, Paremain GP. Incidental abdominal aortic aneurysm on lumbosacral magnetic resonance imaging - a case series. Magn Reson Imaging 2010; 28: 455-7.

5. Fishman SM, Ballantyne JC, Rathmell JP. Bonica's Management of Pain. 4th ed. Philadelphia, Lippincott Williams \& Wilkins. 2010, pp 1099-100.

6. Takeyachi Y, Yabuki S, Arai I, Midorikawa H, Hoshino S, Chiba K, et al. Changes of low back pain after vascular reconstruction for abdominal aortic aneurysm and high aortic occlusion: a retrospective study. Surg Neurol 2006; 66: 172-6.

7. Hirsch AT, Haskal ZJ, Hertzer NR, Bakal CW, Creager MA, Halperin JL, et al. ACC/AHA 2005 Practice Guidelines for the management of patients with peripheral arterial disease (lower extremity, renal, mesenteric, and abdominal aortic): a collaborative report from the American Association for Vascular Surgery/Society for Vascular Surgery, Society for Cardiovascular Angiography and Interventions, Society for Vascular Medicine and Biology, Society of Interventional Radiology, and the ACC/AHA Task Force on Practice Guidelines (Writing Committee to Develop Guidelines for the Management of Patients With Peripheral Arterial Disease): endorsed by the American Association of Cardiovascular and Pulmonary Rehabilitation; National Heart, Lung, and Blood Institute; Society for Vascular Nursing; TransAtlantic Inter-Society Consensus; and Vascular Disease Foundation. Circulation 2006; 113: e463-654.

8. Singh K, Bønaa KH, Jacobsen BK, Bjørk L, Solberg S. Prevalence of and risk factors for abdominal aortic aneurysms in a population-based study : The Tromso Study. Am J Epidemiol 2001; 154: 236-44.

9. Forsdahl SH, Singh K, Solberg S, Jacobsen BK. Risk factors for abdominal aortic aneurysms: a 7-year prospective study: the Tromso Study, 1994-2001. Circulation 2009; 119: 2202-8.

10. Greco G, Egorova NN, Gelijns AC, Moskowitz AJ, Manganaro AJ, Zwolak RM, et al. Development of a novel scoring tool for the identification of large $>/=5 \mathrm{~cm}$ abdominal aortic aneurysms. Ann Surg 2010; 252: 675-82.

11. Brewster DC, Cronenwett JL, Hallett JW Jr, Johnston KW, Krupski WC, Matsumura JS. Guidelines for the treatment of abdominal aortic aneurysms. Report of a subcommittee of the Joint Council of the American Association for Vascular Surgery and Society for Vascular Surgery. J Vasc Surg 2003; 37: 1106-17.

12. Gouliamos AD, Tsiganis T, Dimakakos P, Vlahos LJ. Screening for abdominal aortic aneurysms during routine lumbar CT scan: modification of the standard technique. Clin Imaging 2004; 28: 353-5.

13. Brewster DC, Darling RC, Raines JK, Sarno R, O'Donnell TF, Ezpeleta M, et al. Assessment of abdominal aortic aneurysm size. Circulation 1977; 56(3 Suppl): II164-9.

14. Scott RA, Ashton HA, Kay DN. Abdominal aortic aneurysm in 4237 screened patients: prevalence, development and management over 6 years. Br J Surg 1991; 78: 1122-5.

15. Boll AP, Verbeek AL, van de Lisdonk EH, van der Vliet JA. High prevalence of abdominal aortic aneurysm in a primary care screening programme. Br J Surg 1998; 85: 1090-4. 